\title{
HACIA UNA HISTORIA CRÍTICA DEL ARTE PALEOLÍTICO: LA HISTORIA SOCIAL DE LAS CIENCIAS SOCIALES COMO PARADIGMA
}

\author{
Towards a critical history of Palaeolithic art: \\ The social history of social science as paradigm
}

\author{
Oscar Moro Abadía y Manuel R. González Morales* \\ Recibido el 23 de julio de 2007. Aceptado el 30 de agosto de 2007.
}

Resumen. Algunos trabajos recientes han subrayado la emergencia, durante los años noventa, de una historia crítica de la arqueología opuesta a la historiografía tradicional. Como estos autores señalan, el principal objetivo de la nueva generación de historiadores es que la historia de la arqueología asuma una posición más central en el conjunto de la disciplina. En este contexto, pensamos que el proyecto de la historia social de las ciencias sociales de Pierre Bourdieu puede proporcionar un modelo útil para construir una historiografía crítica que introduzca el pasado en los debates disciplinares. Tomando como ejemplo nuestro reciente trabajo sobre arte paleolítico, sugerimos aqui algunas ideas sobre la utilización de la historia social de Bourdieu como instrumento de análisis histórico que permita comprender mejor los cimientos de la ciencia arqueológica.

Palabras clave: Historia de la arqueología. Historia social de las ciencias sociales. Bourdieu. Reflexividad. Arte paleolítico.

Abstract. Recent works on archaeological theory have emphasized the emergence during the nineties of a critical history of archaeology as opposed to a traditional historiography. As these authors have pointed out, the main aim of the former is that the history of archaeology assumes a more central position in the discipline. In this context, we suggest that Pierre Bourdieu's project of Social history of social science can provide archaeologists with a useful model to build a critical historiography of the discipline that articulates the past with contemporary debates on archaeological knowledge. Taking our recent works on Paleolithic art into account, we propose in this paper some critical thoughts on Bourdieu's Social History as an instrument of historical analysis that is useful to better understand the foundations of archaeological science.

Key Words: History of Archaeology. Social history of social science. Bourdieu. Reflexivity, Paleolithic Art.

(*) Oscar Moro Abadía: Becario postdoctoral. Instituto Internacional de Investigaciones Prehistóricas de Cantabria. Edificio Interfacultativo. Avenida de los Castros S/N, 39005 Santander (Cantabria) y Department of Anthropology. University of Toronto. 100 St. Georges Street. Toronto. Notario. M5S $3 \mathrm{G} 3$.

Manuel R. GonzÁlez Morales: Catedrático de Prehistoria. Instituto Internacional de Investigaciones Prehistóricas de Cantabria. Edificio Interfacultativo. Avenida de los Castros S/N, 39005 Santander (Cantabria). 


\section{HACIA UNA HISTORIA CRÍTICA DE LA ARQUEOLOGÍA}

"La historia de la arqueología ha dejado de ser vista como marginal para la arqueología y está asumiendo una posición más central en la disciplina [...] Por consiguiente, la historia de la arqueología está comenzando a jugar un papel principal en la comprensión y en la aplicación del conocimiento arqueológico" (Trigger, 2001: 639).

Como señalaba Bruce G. Trigger, la historia de la arqueología ha adquirido un protagonismo importante durante los últimos años. Pese a que todavía es una actividad secundaria para muchos arqueólogos, lo cierto es que puede hablarse de un boom historiográfico que ha tenido su reflejo en la publicación de numerosos trabajos sobre la relación entre arqueología, identidad, género o nacionalismo (Silberman, 1989; Kohl y Fawcett, 1995; Díaz-Andreu y Champion, 1996; Atkinson y Banks y O'Sullivan, 1996; Jones, 1997; Díaz-Andreu y Sorensen, 1998; Härke, 2000; Díaz-Andreu, 2002), la reciente aparición de varias síntesis generales (Trigger, 1989; Robertshaw, 1990; Schnapp, 1993; Coye, 1997; Gran-Aymerich, 1998; Kehoe, 1998; Murria, 1999; Murria, 2001, Díaz-Andreu, 2002) o la institucionalización de la disciplina a través de la puesta en marcha de proyectos como AREA (Archives for European Archaeology) dirigido por Alain Schnapp y Nathan Schlanger.

Este "despertar historiográfico" (Díaz-Andreu, 2002: $35)$ ha sido posible gracias a una nueva reflexión a propósito de los objetivos, los métodos y la función de la historia de la arqueología. Como han señalado varios autores (Fahnestock, 1984; Pinsky, 1989b; Richard, 1991: IV), el reciente auge de la disciplina está relacionado con la sustitución de la "historia-memoria" por la "historia-critica" (Gran-Aymerich, 1998: 12). Efectivamente, hasta fecha muy reciente, la historia de la arqueología se habia identificado casi totalmente con una whig history cuya función era mostrar el inevitable triunfo de la ciencia positiva sobre otros modos de conocimiento. Sin embargo, a partir de los años noventa los historiadores reaccionaron contra la historia positivista y de legitimación (Richard, 1991: IV; Richard, 1993: 11; Groenen, 1994: 18; Coye, 1997: 7; Schlanger, 2002: 128) subrayando "la necesidad acuciante [de construir] una historiografía crítica de la disciplina que articule el pasado directamente con los intereses filosóficos y teóricos contemporáneos y futuros" (Pinsky, 1989: 88). Como consecuencia de este cambio de enfoque, la historia disciplinar ha pasado de ser la "ocupación accidental de un limitado número de investigadores" (Meltzer, 1989: 10) a ser consideraba una herramienta importante para comprender el trabajo de los arqueólogos. De hecho, la mayoría de ellos consideran que "ignorar el contexto histórico de los mo- dernos argumentos metodológicos y teóricos [...] es una estupidez" (Sabloff, 1989: 35).

Para comprender las características fundamentales de la nueva historia de la arqueología, podemos repasar brevemente la obra de Bruce G. Trigger, el más reputado de los arqueólogos- historiadores. En 1985, Trigger asignaba un doble cometido a la historia de la arqueología: Por un lado, mostrar la influencia del descubrimiento de datos y técnicas en nuestra comprensión del pasado, por otro, ofrecer un marco de referencia que permita comprender la multiplicidad de factores que influyen en la interpretación de los datos arqueológicos (Trigger, 1985: 233). En 1989, Trigger consideraba que la disciplina tenía que ayudar a determinar si nuestro conocimiento sobre el pasado ha progresado verdaderamente: "En el examen de los sucesivos modelos que han influido en la interpretación de los datos arqueológicos, intentaré determinar hasta que punto la comprensión del comportamiento y de la historia humana ha sido alterada irreversiblemente como resultado de la actividad arqueológica" (Trigger, 1989, 35). En definitiva, mostrando la correspondencia factual entre la interpretación arqueológica y un conjunto de datos cada vez mayor, la historia de la arqueología tendría algo que decir sobre el problema de la objetividad del conocimiento. En 2001, Trigger insistía en subrayar la utilidad epistemológica de la historia al afirmar que "el campo [la historia de la arqueología] también proporciona una base para discutir problemas epistemológicos en términos familiares para el arqueólogo" (Trigger, 2001: 639). Como Trigger, numerosos compañeros han defendido la necesidad de construir una historia crítica de la arqueología que se convierta en una herramienta importante para comprender la práctica arqueológica. Así por ejemplo, para Murray explorar nuevas historias de la arqueología "puede ayudarnos a comprender cómo el edificio de la moderna arqueología -sus agendas, conceptos, categorias, patrones de socialización, e instituciones- Ilegó a establecerse" (Murria, T., 2002: 238), Pinsky defiende que la historia de la arqueología puede jugar un papel importante en la actual (y futura) dirección de la práctica arqueológica (Pinsky, 1989b: 88), o Susan Bender considera la historia de la arqueología que nos ayuda a comprender nuestras raices intelectuales (Bender, 1989: 55).

En líneas generales, es evidente que la historia de la arqueologia ha experimentado cambios importantes a lo largo de los últimos años. Dichos cambios están relacionados con la aparición, a principios de los años ochenta, de una reflexión en torno a la naturaleza de la disciplina. Sólo cuando la historia de la arqueología se constituyó como objeto de estudio para arqueólogos e historiadores, una historia crítica fue posible. Resumiendo, hasta 1980 los arqueólogos escriben historias de la arqueología (en plural), pero sólo a partir de entonces reflexionan sobre el significado de la historia de la arqueología (en singular). Sin embargo, conviene ser cautos a 
la hora de valorar los logros de esta nueva historia de la arqueología. Por un lado, el paradigma dominante hasta los años ochenta, la historia presentista, ha sido criticado pero no ha sido sustituido. Trabajos como los de Natalie Richard (1991), Paul Bahn (1996), Marc Groenen (1994), William Stiebing (1993) o Eve Gran-Aymerich (1997) atestiguan una cierta continuidad de la whig history of archaeology.' Por otro lado, la historia de la arqueología continúa permaneciendo ajena a los principales debates en historia y en historia de la ciencia. Esto ha provocado que, pese a que la mayoria de los nuevos historiadores insisten una y otra vez en la necesidad de sustituir el paradigma presentista dominante, la propuesta de nuevos modelos teóricos haya sido limitada.

Nuestra idea fundamental es que la historia de la arqueología sólo se convertirá en una historia verdaderamente crítica (i. e. que reflexiona sobre sí misma) y útil (i. e. que pueda jugar un papel importante en la comprensión de la arqueología científica) cuando sea capaz de integrar los debates, modelos y teorías provenientes de la historia y de la historia de la ciencia. Por ello, queremos explorar en este artículo la historia social de las ciencias sociales de Pierre Bourdieu como paradigma para constituir una historia critica del arte paleolítico. En este sentido, en la primera parte de este trabajo presentamos las líneas generales del pensamiento del sociólogo francés, centrándonos en aquellas ideas que pueden sernos de utilidad. En la segunda parte del artículo intentaremos demostrar la utilidad de dicho modelo en un caso concreto: la historia del arte paleolítico. Repasando los trabajos que desde hace algunos años estamos llevando a cabo a propósito de los conceptos de arte mobiliar y de arte parietal paleolítico, intentaremos demostrar cómo el modelo bourdieusiano puede convertirse en una herramienta válida para la constitución de una historia crítica de la arqueología $y$, de manera más general, para una historia crítica de las ciencias sociales.

\section{LA HISTORIA SOCIAL DE LAS CIENCIAS SOCIALES DE PIERRE BOURDIEU}

La obra del sociólogo francés Pierre Bourdieu (19302002) se ha convertido en una referencia esencial para comprender las ciencias sociales en los inicios del siglo XXI. La heterogeneidad de sus investigaciones (que incluyen desde el arte hasta el sistema escolar, pasando por el lenguaje, la televisión o la economía), su innegable compromiso político y la brillantez teórica de sus escritos lo han convertido en una de las figuras fundamentales del pensamiento francés contemporáneo. No es nuestra intención valorar aqui a un autor que ha merecido numerosos e interesantes análisis (Calhoun, et al., 1993; Lahire, 1999; Lane, 2000; Mounier, 2001; Alonso, et al., 2004). Simplemente, intentaremos hacernos eco de uno de sus proyectos fundamentales, la historia social de las ciencias sociales, y de sus posibilidades de aplicación para la construcción de una historia crítica de la arqueología.

Para comprender la historia social de las ciencias sociales de Bourdieu comenzaremos con una reflexión general a propósito del significado y la función de la historia en la obra del autor. Aunque un trabajo tan heterogéneo como el suyo es susceptible de ser analizado desde varios puntos de vista, pensamos que su trayectoria puede ser interpretada como una lucha contra lo que el propio autor denominaba la deshistorización (Bourdieu, 1992 : 393), la ilusión de lo absoluto (Bourdieu, 1992: 394), el privilegio de lo universal (Bourdieu, 1994: 223), la amnesia de la génesis (Bourdieu, 2000:16) o, en definitiva, contra el olvido de la historia (Bourdieu, 1997: 54). En este sentido, su obra puede describirse como un proyecto de historización radical, es decir, como "una voluntad de llevar a sus límites la historización del objeto de estudio" (Vázquez, 2004) frente a los procesos de naturalización de dicho objeto. En nuestra opinión, esta última cuestión, "la transformación de la historia en naturaleza" (fórmula que Bourdieu toma prestada de Lukács, 1923), es el punto de partida de varios de sus trabajos más importantes. Desde luego, esta interpretación no pretende ser tanto un lectura encaminada a justificar nuestro propio trabajo, cuanto una constatación de lo qué el propio sociólogo no se cansa de repetir. Es suficiente con darle la palabra para comprobarlo.

En Les règles de l'art (Bourdieu, 1992), Bourdieu propone un "análisis científico de las condiciones sociales de la producción y de la recepción de la obra de arte" (Bourdieu, 1992: 14). Frente a la idea del universo artístico como un mundo que escapa indefinidamente a cualquier explicación, Bourdieu se propone mostrar las condiciones socio-históricas de producción de la obra de arte mediante las reglas del análisis sociológico. Para ello, comienza planteando el problema en los siguientes términos: ¿Por qué se confiere al arte un estatuto de excepción? (Bourdieu, 1992: 11) ¿Por qué la idea tan extendida de que al análisis científico destruye la experiencia artística, la más elevada que el hombre pueda conocer? (Bourdieu, 1992: 9) ¿Por qué el arte se vive como una experiencia absoluta ajena a las contingencias de la génesis? (Bourdieu, 1992: 14). Según Bourdieu, la idea del arte como una experiencia universal está en relación con la deshistorización tanto de la obra de arte como de la mirada sobre dicha obra (Bourdieu, 1992: 393). Según el sociólogo francés, el discurso estético se construye sobre el silencio consciente de las condiciones históricas y sociales de producción de la

(1) Aunque existen excepciones notables (Schnapp, 1993, Coye, 1997, Kaeser, 2004), lo cierto es que la mayoria de los trabajos que adoptan la longue durée continúan reproduciendo un modelo Whig, tal y como intenté demostrar en una comunicación titulada "The War Against the Whigs. Victory...or Defeat?' (Moro Abadia, 0. 2004) presentada en el último congreso de AREA celebrado en Goteborg en 2004. 
obra artística (Bourdieu, 1992: 394). Al negar su historicidad, dicho discurso afirma la trascendencia de la obra de arte $y_{1}$ con ello, el privilegio de quienes saben reconocer, juzgar y valorar dicha trascendencia. Es decir, el privilegio de los estetas. Frente a la deshistorización, Bourdieu propone un análisis científico de las obras de arte que se apoya en dos ideas: En primer lugar, que el arte tiene su historia particular, diferente de la historia economicista del marxismo a lo Kautsky o del expresivismo a la Lukács. En segundo lugar, la idea de que la sociología del arte no sólo no destruye el goce estético sino que lo incrementa (al aumentar la comprensión).

En Méditations pascaliennes, Bourdieu vuelve a cargar contra "el olvido de la historia" (Bourdieu, 1997: 54), centrándose esta vez en el campo filosófico. El libro es una crítica de la canonización escolástica del discurso filosófico y de la resistencia que los filósofos oponen a las ciencias sociales. Tal y como sucedia con el mundo artístico, el privilegio del universo filosófico se construye sobre una eternización (Bourdieu, 1997: 54) de los textos canónicos de la filosofía, considerados portadores de verdades trascendentales e irreductibles al análisis histórico. De este modo, el problema vuelve a situarse en "el rechazo del pensamiento de la génesis" (Bourdieu, 1997: 54) y en negarse a aceptar el discurso filosófico tal y como es, es decir, como un producto de la historia (Bourdieu, 1997: 137). Esa lectura deshistorizante (Bourdieu, 1997: 54) tiene como objetivo asegurar el privilegio de los garantes del pensamiento escolástico. Otro ejemplo. En su libro La domination masculine (1998), Bourdieu intenta determinar las claves de dicha dominación. ¿Por qué la dominación masculina sobre la mujer se consolidó como una estructura social? ¿Por qué dicha autoridad se mantiene intacta aún cuando conocemos muchos de los mecanismos en los que se apoya? En opinión de Bourdieu, la clave es que dicha dominación se ejerce a través de una forma particular de violencia simbólica (Bourdieu, P., 1998: 39) que consiste en que tanto los dominadores (hombres) como los dominados (mujeres) aceptan como natural el orden de las cosas en el que dicha dominación se inscribe. En otras palabras, la clave se encuentra en la deshistoricización y en la eternización relativas de las estructuras de la división sexual, que han pasado a ser consideradas naturales cuando, en realidad, son pura historia.

Los análisis del campo artístico, el campo filosófico y de la dominación masculina constituyen tres buenos ejemplos de la lucha de Pierre Bourdieu contra la deshistorización. Desde luego, no son los únicos. Sus trabajos contra el privilegio de lo universal en el punto de vista escolástico (Bourdieu, 1994: 223), contra la visión antihistórica de una ciencia económica construida sobre la amnesia de la génesis (Bourdieu, 2000: 16) o contra la ilusión de la neutralidad del campo científico (Bourdieu, 2001) muestran que estamos ante una de las preocupaciones recurrentes del pensador francés.
La interpretación de la lucha contra la deshistorización como uno de los vectores directores de la obra de Bourdieu nos introduce en una de sus ideas fundamentales a propósito de la historia: "Me he esforzado en mostrar que aquello que llamamos "lo social" es de parte a parte historia. La historia está inscrita en las cosas, es decir, en las instituciones (las máquinas, los instrumentos, el derecho, las teorias científicas, etc.) y también en el cuerpo. Todo mi esfuerzo está encaminado a descubrir la historia allí donde se esconde mejor, en los cerebros y en los pliegues del cuerpo. El inconsciente es la historia [...] Panofsky recuerda que cuando alguien levanta su sombrero para saludar, reproduce sin saberlo el gesto mediante el cual los caballeros, en la Edad Media, levantaban su casco para manifestar sus intenciones pacíficas [...] (Bourdieu, 1980b: 74). La idea de Durkheim, que Bourdieu gustaba tanto de repetir ("el inconsciente es el olvido de la historia", Bourdieu, 1980b: 81), es fundamental para comprender su proyecto de una historia social de las ciencias sociales. Para Bourdieu, la historia se inscribe de manera inconsciente tanto en el habitus como en el campo:

- El habitus o sistemas de disposiciones que condiciona a los agentes a actuar y a reaccionar de un determinado modo (Bourdieu, 1980: 88- 89) es la historia hecha cuerpo (Bourdieu, 1997: 179). En tanto que signum social (Bourdieu, 1962: 100) las disposiciones que conforman el habitus o la hexis engendran maneras de hablar, de sentir y de pensar en los individuos (Bourdieu, 1980: 117) que no son naturales sino producto de la historia (Bourdieu, 1980: 91). El habitus "sintetiza corporalmente los efectos en la existencia individual de la división social y sexual del trabajo" (Moreno Pestaña, 2004: 150). En consecuencia, el habitus es naturaleza social [e históricamente] constituida (Bourdieu, 1987: 21).

- El campo, o cada uno de los contextos sociales específicos en los que los individuos actúan determinados por la distribución de recursos o de "capital" (Bourdieu, 1980b: 113-120), es la historia objetivada e incorporada en las instituciones (Bourdieu, 1980: 95).

Por lo tanto, la historia se transforma en inconsciente social a través de la constitución del habitus y de los distintos campos sociales (artístico, filosófico, educativo, etc.): "El insconsciente es la historia; la historia colectiva que ha producido nuestras categorías de pensamiento y la historia individual a través de la cual dichas categorias nos son inculcadas" (Bourdieu, 1997: 21). Es precisamente aquí donde entra en juego la historia social de las ciencias sociales. Para Bourdieu, la historia de los distintos campos sociales se ha transformado en el inconsciente de dichos campos, es decir, en el conjunto de reglas, disposiciones, prácticas, teorias, experiencias que rigen el funcionamiento de cada uno de los campos pero que, por evidentes, son invisibles para sus miembros. Como señala a propósito del campo po- 
lítico: "Hay una génesis del campo político, una historia social del nacimiento del campo político. Las cosas que nos parecen evidentes (por ejemplo, la elección por mayoría) son el producto de invenciones históricas extremadamente largas. Las cosas que parecen haber existido toda la eternidad son a menudo una invención reciente" (Bourdieu, 2000a: 53) ¿Por qué los principios de estructuración que constituyen cualquier campo acaban por convertirse en invisibles para sus agentes? Porque como Bourdieu sugiere, la relación rutinaria con la herencia, convertida en doxa disciplinaria, provoca casi inevitablemente la amnesia de la génesis (Bourdieu, 1995: 111). Tomando como ejemplo el caso de la arqueología, en su práctica diaria los arqueólogos ponen en juego conceptos, ideas, procedimientos, usos, etc. que han conformado el campo y que nos parecen perfectamente evidentes. La puesta en práctica, todos los dias y durante un tiempo prolongado, de dichas disposiciones ha provocado que los arqueólogos olviden, como no podría ser de otro modo, que se trata de disposiciones "enteramente salidas de la historia" (Bourdieu, 1997: 137).

Por consiguiente, el objetivo de la historia social de las ciencias sociales es sacar a la luz ese "inconsciente" que rige los distintos campos. Es por ello que Bourdieu define su empresa como una "ciencia del inconsciente" (Bourdieu, 1982 : 10): "La historia social de las ciencias sociales no es una ciencia como cualquier otra [...] Extendiendo el conocimiento histórico y sociológico del pasado y del presente de la sociología y de la historia a su dimensión institucional, [la historia social de las ciencias sociales] proporciona a la historia y a la sociología un medio de explorar y de conocer su inconsciente más específico, que está inscrito en su cerebro, a través de la experiencia presente y pasada de la disciplina" (Bourdieu, 2004: 19). En el caso del campo científico, el objetivo es por tanto "actualizar los presupuestos que están inscritos en el principio mismo de las empresas científicas del pasado y que perpetúa, frecuentemente en estado implícito, la herencia científica colectiva, problemas, conceptos, métodos o técnicas" (Bourdieu, 1995: 111).

Sin embargo, queda por responder una cuestión fundamental. Aún aceptando que Bourdieu tenga razón en que las reglas que rigen la práctica científica sean históricas y no naturales (algo que parece fuera de toda duda) ¿Para qué mostrar su historicidad? ¿Qué gana la ciencia con esta operación? ¿Acaso no le estamos haciendo el juego al relativismo al afirmar que "la verdad científica reside en una especie particular de condiciones sociales de producción" (Bourdieu, 1975: 91; Bourdieu, 1976: 75)? Es el propio Bourdieu quien plantea estas dudas: "¿Puede sobrevivir la verdad a una historización radical? [...] ¿Acaso el historicismo radical [...] no conduce a destruir la idea misma de verdad, destruyéndose de este modo a sí mismo?" (Bourdieu, 2001: 11). Para responder a estas cuestiones es necesario aclarar que Bourdieu no puede, en ningún caso, englobarse dentro del "post-modernismo". Como el propio autor repite una y otra vez, su proyecto no está encaminado a relativizar el conocimiento científico, sino a reforzarlo (Bourdieu, 1992b: 167; Bourdieu, 2001: 8). Y a reforzarlo tanto contra los excesos del universalismo abstracto como contra ciertos delirios "postmodernos" que pretenden socavar la confianza en la ciencia y, especialmente, en las ciencias sociales (Bourdieu, 2001: 5-6). En este sentido, su proyecto prolonga el eco de la razón autocrítica de Kant, al proponer una Realpolitik de la razón (Bourdieu, 1992b: 150-174; Bourdieu, 1995: 125) o una "Aufklärung permanente de la Aufklärung" (Bourdieu, 1997: 86): "No hay contradicción, pese a las apariencias, en luchar al mismo tiempo contra la hipocresía mistificadora del universalismo abstracto y a favor del acceso universal a las condiciones de acceso a lo universal, objetivo primordial de todo verdadero humanismo que la predicación universalista y la (falsa) subversión nihilista tiene en común olvidar" (Bourdieu, 1997: 86).

Para comprender en qué sentido la historia social de las ciencias sociales puede servir al progreso de la ciencia, es necesario examinar la idea bourdieusiana de "reflexividad", concepto con una larga tradición en historia de la ciencia (Bloor, 1973: 39- 40) y al que Bourdieu le dio un sentido bastante concreto. Como Francisco Vázquez ha señalado, pueden distinguirse tres etapas en el uso que Bourdieu hace de este término. Un primer momento, que va desde Le métier $d u$ sociologue (Bourdieu, y Chamboredon, y Passeron, 1968) hasta Le sens pratique (Bourdieu, 1980), en el que Bourdieu define la reflexividad como "una autoconciencia de los presupuestos epistemológicos empeñados en la práctica de la investigación" (Vázquez, 2004: 354). Una segunda fase, durante los años ochenta, en la que Bourdieu concibe la reflexividad como el mecanismo que permite acceder al universo inconsciente del investigador social, permitiéndole de este modo conocer los determinismos que condicionan su trabajo. Una tercera fase, a partir de los años ochenta, donde la reflexividad es presentada como un requisito, no sólo metodológico sino también ético, para la construcción del trabajo científico. Aunque las tres dimensiones son fundamentales, nos centraremos ahora en la segunda para explicar la utilidad que el proyecto de Bourdieu puede tener para la propia ciencia.

El trabajo sobre el inconsciente propuesto por la historia social de las ciencias sociales constituye una parte esencial del imperativo de reflexividad sobre el que debería reposar toda empresa científica. Como el propio Bourdieu señala, "la reflexividad es un instrumento para producir más ciencia, no para destruir la posibilidad de dicha ciencia: no intenta descorazonar la ambición científica sino hacerla más realista [...]" (Bourdieu, 1992b: 167) Conocer las condiciones sociohistóricas en las que se ha producido el conocimiento significa dar acceso a los propios científicos a los mecanismos sociales que condicionan su práctica. Esto es fundamental por dos razones: En primer lugar, porque la reflexividad "fa- 
vorece el progreso del conocimiento de los coerciones sociales que pesan sobre el conocimiento, haciendo posible una política más responsable tanto en lo ciencia como en la política" (Bourdieu, 1992b: 167- 168). En otras palabras, sólo a través de un análisis histórico del inconsciente colectivo podremos determinar los condicionantes sociológicos que influyen en la construcción del conocimiento $y$, de este modo, podremos trabajar para superarlos. En segundo lugar, la reflexividad es fundamental porque permite "liberar a los intelectuales de sus ilusiones y, en primer lugar, de la ilusión de no tener ilusiones" (Bourdieu, 1992b: 168). Sólo a través de la reflexividad podremos destruir muchos de los mitos que permanecen anclados en nuestro inconsciente colectivo, convirtiéndonos, de este modo, en señores de nuestro pensamiento. Aunque esa empresa comporta riesgos, no debemos olvidar que "sólo el pensamiento que se hace violencia a sí mismo es lo suficientemente duro para quebrar mitos" (Horkheimer y Adorno, 1944: 60).

\section{HACIA UNA HISTORIA SOCIAL DE LA ARQUEOLOGÍA: EL EJEMPLO DEL ARTE PALEOLÍTICO}

En el anterior epígrafe hemos presentado las líneas generales de la historia social de las ciencias sociales de Pierre Bourdieu. Quisiéramos mostrar ahora cómo dicha teoría puede convertirse en una marco teórico de referencia para la construcción de una historia crítica de la arqueología. Al fin y al cabo, como señalaba Gilles Deleuze, "una teoría es exactamente como una caja de herramientas [...] es preciso que sirva, que funcione, y que funcione para otros, no para uno mismo. Si no hay personas que se sirvan de ella, comenzando por el propio teórico, que deja entonces de ser teórico, es que la teoría no vale nada, o que aún no llegó su momento" (Foucault, 1972: 107-108). Para ello, mostraremos cómo hemos aplicado dichas ideas y conceptos a nuestra investigación a propósito del arte paleolítico.

\subsection{En el inconsciente del arte paleolítico: los conceptos de "arte mobiliar" y de "arte parietal"}

Con motivo del centenario de la publicación de Mea culpa d'un sceptique (Cartailhac, 1902), comenzamos a estudiar el problema del reconocimiento del arte parietal paleolítico. Aunque el rechazo de la autenticidad de Altamira había sido objeto de numerosas publicaciones, entendiamos que era conveniente revisar muchos de los lugares comunes a propósito de esta cuestión. Ese trabajo nos obligó a profundizar en los dos conceptos que han estructurado nuestra moderna comprensión del arte paleolítico: el "arte mobiliar paleolítico" y el "arte parietal paleolítico".
Según una opinión muy extendida, "el arte rupestre [0 parietal] se sitúa en las paredes (en latín paries) de las cuevas y abrigos, extendiéndose la noción de paredes a los suelos y techos. Por el contrario, el arte mobiliar, móvil por definición, está representado por objetos portátiles o más exactamente, que no están fijos o sujetos de manera alguna a una estructura inmobiliaria definida" (Delporte, 1990: 32). De acuerdo con Ann Sieveking, "el arte paleolítico [...] ha sido clasificado por los escritores del siglo XX en dos categorías; arte mural y arte de las pequeñas piezas portátiles encontradas en lugares de habitación. Esta segunda categoría se denomina en francés "art mobilier". Es dudoso que sus creadores reconocieran tal diferencia [...] pero es una división conveniente para el estudio" (Sieveking, 1979: 7- 8). Efectivamente, la distinción entre "arte mobiliar" y "arte parietal" es el punto de partida de la mayoría de las interpretaciones a propósito del arte paleolítico.

Esta última afirmación no significa que dicha división haya sido aceptada sin discusión. En primer lugar, descubrimientos importantes realizados durante los últimos años han obligado a los especialistas a redefinir el concepto de "arte parietal paleolítico" (tradicionalmente asimilado al arte encontrado en las paredes de las cuevas y abrigos). Así, hace aproximadamente una década, el arte parietal fue descubierto por primera vez en yacimientos al aire libre en Europa. Ejemplos como el de Foz Côa mostraron entonces la existencia de un "cave art without caves" (Bahn, 1995: 231). Como resultado de este proceso, los arqueólogos han comenzado a sustituir el término tradicional de "arte parietal" por el de "rock art" ("I'art des parois" en la literatura francesa). En segundo lugar, varios autores han mostrado los inconvenientes de dividir el arte paleolítico en mobiliar y parietal (Leroi Gourhan, 1971: 44; Lorblanchet, 1995: 13). Así, por ejemplo, Denis Vialou se preguntaba: “¿Cómo es posible que esas interpretaciones generales y generalizantes separen (casi siempre) el arte mobiliar del arte parietal? ¿Se puede de verdad pensar en desarrollar interpretaciones sobre los signos, la magia, los estados de alteración de la conciencia, la sexualidad o las figuraciones femeninas en el Magdaleniense teniendo solamente en cuenta las interpretaciones parietales?" (Vialou, 1998: 269).

A pesar de estas críticas, es suficiente con un repaso general de algunas de las obras de referencia de la disciplina (Laming- Emperaire, 1962: 21; Ucko y Rosenfeld, 1967: 8; Brézillon, 1969: 35; Bray y Trump, 1970: 51; Barandiarán, 1972, Delporte, 1981: 14; Whitehouse, 1983: 331-332; Leroi-Gourhan, 1988: 70; Delporte, 1990: 32; Bahn, 1992: 378, Lorblanchet, 1995: 13 y 21) para constatar que dichas interpretaciones toman como punto de partida la división entre "arte mobiliar" y "arte parietal". De este modo, los dos conceptos se han convertido en el sustrato que subyace a cualquier aproximación a la significación del fenómeno artístico en el Paleolítico. Para la mayoría de los arqueólogos, 
esta dicotomía se impone como algo evidente, "sin ninguna justificación ni crítica analítica de esta fractura del conjunto del Ilamado arte paleolítico" (Vialou, 1998: 269). Incluso aquellos que, como Denis Vialou, son conscientes de los problemas de esta clasificación, siguen utilizando dicho aparato conceptual en su trabajo práctico (Vialou, 2001: 2001). Como ha señalado Richard Bradley a propósito del arte parietal, "el término es insatisfactorio, pero, como sucede con muchos conceptos técnicos, es demasiado tarde para buscar una alternativa" (Bradley, 1997: 4-5).

En definitiva, la división entre "arte mobiliar" y "arte parietal" se ha inscrito en el inconsciente disciplinar a través de un largo proceso de deshistorización. En primer lugar, su utilización recurrente ha provocado que haya pasado a considerarse una distinción evidente y que los arqueólogos no se vean obligados a justificar su uso. Cuando un prehistoriador analiza la tecnología del "arte mobiliar" (D'Errico, 1988), la arquitectura del "arte parietal paleolítico" (Vialou, 2001) o la gramática del "arte mobiliar" (Sauvet y Wlodarcyk, 1995) no comienza su investigación con una explicación metodológica que justifique el empleo de dichas categorias: se da por sentado que se trata de un procedimiento natural y evidente. La división entre "lo parietal" y "lo mobiliar" forma parte del habitus del prehistoriador: no se trata de un procedimiento consciente conforme a las reglas del razonamiento científico y el método experimental, sino de un "sentido práctico", un modus operandi compartido por todos los miembros del campo. En segundo lugar, el uso prolongado de esta clasificación ha tenido como consecuencia lo que Bourdieu denomina el olvido de la génesis: pese a tratarse de los dos conceptos más utilizados de la disciplina, no existe ningún trabajo sobre su formación histórica. En otras palabras, nadie se ha visto en la obligación de recordar (porque nadie se acuerda) que se trata de dos conceptos históricos. Esto ha provocado la naturalización de la división que estos dos conceptos instauran, descrita a menudo como una división objetiva y lógica de las obras de arte según el soporte en el que fueron realizadas: "Las diferentes manifestaciones de lo que llamamos arte aparecen por primera vez en el Paleolítico superior. Se dividen, para su estudio, según el soporte sobre que fueron realizadas. Se denomina arte rupestre o parietal al realizado sobre las paredes de cuevas o abrigos, y arte mueble o mobiliar a los pequeños objetos transportables realizados sobre piedra, asta, hueso o marfil." (Menéndez y Jimeno y Fernández, 1997: 40).

En modo alguno pretendemos culpar a los arqueólogos de este olvido de la historia que, en cierto sentido, es un proceso inherente a la propia práctica científica. Es por ello que corresponde a la historia de la ciencia, constituida en una conciencia epistemológica de la disciplina, dar cuenta de esa deshistorización. Es ella quien debe mostrar, y esta es nuestra hipótesis, que las categorías "arte mobiliar" y "arte parietal" no remiten a una clasificación objetiva de las obras de arte paleolítico a partir del soporte sobre el que fueran realizadas, sino a una fractura en la definición del arte paleolítico entre 1860 y 1905.

\subsection{Poniendo en marcha la historia: Una genealogía de los conceptos "arte mobiliar" y "arte parietal"}

Parafraseando a Bourdieu, recordar que lo que, en la práctica científica, aparece como natural es sólo el producto de un prolongado trabajo de naturalización, significa reinsertar en la historia aquellas ideas que han estructurado la ciencia y, por tanto, comprender mejor su significado. Por ello, en este epígrafe proponemos un resumen de la genealogía histórica de los conceptos "arte mobiliar" y "arte parietal" que hemos desarrollado en otros trabajos (Moro Abadía y González Morales, 2003; Moro Abadía y González Morales, 2004).

Para comprender la formación histórica de ambas categorias es necesario situarse en el período comprendido entre 1864 y 1902. Como es conocido, la antigüedad paleolítica del "arte mobiliar" fue aceptada por la comunidad científica internacional hacia 1864, año de publicación del famoso artículo de Lartet y de Chirsty. Por otro lado, aunque las primeras manifestaciones del arte parietal fueron publicadas durante los años ochenta (Sautuola, 1880; Chiron, 1889), su gran antigüedad no fue aceptada hasta inicios del siglo XX (Cartailhac, 1902). El décalage entre los dos momentos de reconocimiento del arte paleolítico (1864 y 1902) es la clave para comprender la formación histórica de la dicotomía que estamos analizando. Para interpretar este proceso es fundamental retener dos ideas:

- En primer lugar, los conceptos "arte mobiliar" y "arte parietal" no existían antes de 1900. Los arqueólogos de finales del siglo XIX no utilizaron dichos términos para describir el arte prehistórico. De hecho, "sólo hacia 1900, se aceptó entre los prehistoriadores la distinción entre el arte parietal y el mueble" (Delporte, 1990: 32)

- En segundo lugar, entre 1860 y 1900 los prehistoriadores elaboraron una definición del arte paleolítico que coincide con lo que hoy en día llamamos "arte mobiliar" y que excluia lo que definimos como "arte parietal".

Teniendo en cuenta estos dos datos, nuestra hipótesis es que lo que durante el siglo XX se ha llamado "arte mobiliar" se corresponde con lo que a finales del siglo XIX se denominaba arte paleolítico y que, por consiguiente, para conocer el significado de dicho concepto es necesario examinar la representación del arte paleolítico entre 1860 y 1900.

En 1864, el descubrimiento de piezas con animales grabados procedentes del Paleolítico en las cuevas de la Vézère provocó una contradicción en el seno de la sociedad europea 
de finales del siglo XIX. En efecto, la aparición de objetos artísticos encajaba muy mal con la imagen, por aquel entonces dominante, de los hombres prehistóricos como salvajes recién salidos de la animalidad: "Si verdaderamente el hombre hizo tales progresos en la concepción del arte sin conocer ni siquiera los metales, es un fenómeno antropológico tan curioso como lo son los propios objetos artísticos, que expresan un grado de lujo engendrado por el ocio y el bienestar" (Falconer, 1864: 630). En realidad, es lógico que la sociedad decimonónica tuviera enormes dificultades para aceptar un arte de esta naturaleza. El último tercio del siglo XIX estuvo dominado por lo que Nathalie Richard ha llamado "el paradigma de la simplicidad" (Richard, 1993: 60). Es decir, por un evolucionismo simple de carácter estrictamente unilineal. Un buen ejemplo son las obras de antropología de esta época que describen la historia de la humanidad como una sucesión unilineal de estadios desde el salvajismo hasta la civilización: Primitive Culture (Tylor, 1871), Ancient Society (Morgan, 1877), Ancient Law (Maine, 1861), Primitive Marriage (McLennan, 1865), The Origin of Civilisation (Lubbock, 1870). En este horizonte de pensamiento, el arte era considerado un símbolo de civilización y, por consiguiente, un elemento exclusivo de las últimas etapas. Nada tiene de extraño, por tanto, que la aparición de pequeños objetos grabados y esculpidos en un contexto paleolítico provocase una contradicción en la sociedad occidental.

En su intento por hacer compatible lo supuestamente incompatible (el arte y lo primitivo), y como no podía ser de otro modo, los arqueólogos utilizaron las categorias que estructuraban su propia visión del arte para construir una representación del arte paleolítico. Dicha visión se corresponde con lo que Kristeller denominó el "sistema moderno del arte", (Kristeller, 1950). Efectivamente, como ha mostrado Larry Shiner, nuestra experiencia artística es una invención reciente, un fenómeno que no tiene ni tres siglos (Shiner, 2001: 3). A partir del siglo XVIII, la moderna definición del arte se consolidó sobre una fractura que se había ido formando lentamente desde el Renacimiento: la separación entre el Arte con mayúsculas ("fine arts", "Beaux-Arts") y la artesanía ("the crafs and popular arts"). En esta época, el Arte (la poesía, la pintura, la arquitectura, la escultura, la música) fue asociado a la inspiración y la genialidad, la trascendencia, la originalidad, la invención, la creación y la inspiración. Por oposición, la artesanía fue asimilada al savoir-faire, la imitación y la reproducción mecánica de un modelo, el trabajo repetitivo, la habilidad técnica desprovista de genialidad y el placer utilitario y ordinario. Este sistema ha estructurado la percepción occidental del fenómeno artístico desde el siglo XVIII hasta nuestros días y es la clave para comprender la definición del arte paleolítico entre 1860 y 1900.

La aparición del arte paleolítico planteó inmediatamente el problema de su clasificación: ¿En qué categoría clasificar las pequeñas estatuillas descubiertas por los arqueólogos?
La cuestión no era sencilla. Por un lado, los europeos tenían que aceptar, por sorprendente que pudiera parecer, que el hombre paleolítico poseía un cierto "amor por el arte, por mínimo que sea" (Lubbock, 1865: 306). En este sentido, era innegable que los prehistóricos poseian un cierto "instinct de luxe" (Lartet y Christy, 1864: 280; Mortillet, 1897: 241). Por otro lado, era impensable conceder el título de "artistas" a unos salvajes que vivían en un estado de civilización tan poco desarrollado. Simplemente, era imposible colocar en el mismo nivel a un Miguel Angel o a un David que a un paleolítico anónimo que desconocía los metales, la domesticación de animales o la agricultura. Para superar esa paradoja, entre 1860 y 1900 los europeos definieron el arte paleolítico (y el arte primitivo en general) a partir de algunos de los valores y de las connotaciones ligados a la artesanía. Es decir, como un arte menor, ingenuo (Mortillet, 1883: 293; Dreyfus, 1888: 224), ligado a los pequeños objetos de decoración personal (Lartet y Christy, 1864: 280; Dupont, 1872: 155; Dreyfus, 1888: 225), ornamental (Lubbock, 1870: 51; Evans, 1872: 448; Wilson, 1862 : 289; Mortillet, 1897: 241), producto de un cierto savoir- faire y reflejo de una gran incapacidad técnica (Mortillet, 1883: 295-297, Dreyfus, 1888: 225).

En este contexto, no es casualidad que la antigüedad paleolítica de las pinturas parietales encontradas en Chabot o en Altamira fuese rechazada hasta los inicios del siglo XX. Las pinturas parietales eran incompatibles con la idea del hombre prehistórico dominante en ese momento: "Entre la vida a oscuras en las cavernas sobre restos de cocina corrompidos y la aspiración a decorar la estancia existe una contradicción tan grande, que explica bien no se haya encontrado hasta ahora en aquellas nada parecido a lo que nos ocupa." (Quiroga y Torres, 1880: 266). Solo a finales del siglo XIX, gracias al debilitamiento del evolucionismo como paradigma dominante y al reconocimiento de un mayor grado de complejidad en las sociedad primitivas, fue posible la aceptación de dichas pinturas como paleolíticas.

Este análisis histórico es fundamental para comprender el verdadero significado de nuestra moderna definición de los conceptos "arte mobiliar" y "arte parietal". Dado, y esto es esencial, que el conjunto de objetos que hoy llamamos "arte mobiliar" coincide con lo que los prehistoriadores de finales entre 1860 y 1900 llamaron "arte paleolítico", es evidente que dicho concepto nos remite a la división entre "arte" y "artesanía" que dichos arqueólogos utilizaron para definir el arte paleolítico. En otras palabras, la historización del "arte mobiliar" y del "arte parietal" nos permite comprender que la definición normativa de dichos conceptos es una ilusión escolástica parcialmente falsa: Lo que dichos conceptos reproducen en realidad es nuestra moderna distinción entre "arte" y "artesanía". Tal y como hemos señalado en otro lugar, "la división entre arte parietal y arte mobiliar ha prevalecido como neutral porque está ligada a una idea que ha estructurado la interpretación occidental del fenómeno artístico desde el siglo XVIII: la 
distinción entre arte y artesanía. Esta división está tan arraigada en nuestras mentes que somos incapaces de verla" (Moro Abadía y González Morales, 2004: 334).

\subsection{Cómo la historia social de la arqueología contribuye al progreso de la arqueología: Reflexiones en torno al caso del arte paleolítico}

La historización de los conceptos "arte parietal" y "arte mobiliar" muestra como dichas categorías remiten, más que a una clasificación de las obras de arte según su soporte, a la transferencia al Paleolítico de nuestra moderna división entre "Arte" y "artesanía". Sin embargo, para que este ejercicio cobre todo su sentido es necesario responder a una cuestión importante: ¿Cómo esta historia social de la arqueología puede contribuir a la mejora de la ciencia? En nuestra opinión, dicha historia se constituye en un arma fundamental para el progreso de la arqueología al introducir la idea de reflexividad en la práctica científica. Dicha reflexividad funciona a dos niveles. En primer lugar, la historia social de la arqueología permite destruir muchos de los mitos sobre los que se apoya la construcción disciplinar. Pensar que la dicotomía parietal- mobiliar reproduce una clasificación neutral del arte paleolítico es una ilusión de la que sólo podemos liberarnos a través de un análisis que muestre la formación histórica de dicha división. La historia social permite, por tanto, liberar a la ciencia del mito ofreciendo a los científicos una compresión más adecuada de los conceptos con los que trabajan.

En segundo lugar, la historia social de ciencia puede contribuir al progreso científico convirtiéndose en una conciencia epistemológica de la arqueología. Para comprender de qué manera la historia social de la arqueología puede constituirse como instrumento de reflexión sobre la naturaleza de la práctica arqueológica, tomaremos como ejemplo un texto de los muchos que proponen una interpretación sobre el significado del arte paleolítico,

"Siguiendo a Wiessner (1983), nosotros también hacemos una distinción entre un "estilo autoafirmativo" (assertive style) y "estilo emblemático" (emblemic style). El arte portátil, principalmente ornamentaciones, probablemente representa el estilo autoafirmativo o "variación formal de la cultura material relacionada con lo personal y que aportan información sobre la identidad individual" (Wiessner, 1983: 258). Este estilo funciona en un nivel idiosincrático para diferenciar unos individuos de los otros. El arte parietal, por otro lado, probablemente expresa lo que Wiessner denomina estilo emblemático o "variación formal de la cultura material que tiene un referente claro y que transmite un mensaje inequívoco a una población determinada" (Wiessner
1983: 258). Por definición el estilo emblemático siempre conlleva una carga simbólica, mientras que el estilo autoafirmativo no necesariamente" (Barton, et al. 1989).

Al tomar como referencia la división entre "arte mobiliar" y "arte parietal", estos autores establece una relación entre dos interpretaciones diferentes. Por un lado, los conceptos "parietal" y "mobiliar" nos remiten a la extrapolación de nuestras modernas categorías de "artesanía" y "arte" a la definición del arte paleolítico. Por otro lado, las ideas de assertive style y de emblemic style definen dicho arte como un sistema de información. Así, para que la hipótesis de estos autores sea epistemológicamente válida será necesario comprobar que la correlación que, implícitamente, se establece entre estas dos interpretaciones no es contradictoria. Lo que este ejemplo indica es que la historia social de la arqueología (que muestra que los conceptos "arte mobiliar" y "arte parietal" suponen, de facto, una interpretación del arte parietal) puede jugar un papel fundamental en el progreso de la ciencia al constituirse en una suerte de conciencia epistemológica que introduzca criterios de valoración objetiva de las hipótesis. De este modo, la historia social de la ciencia contribuirá decisivamente a su progreso.

\section{CONCLUSIÓN}

Tomando como ejemplo los estudios sobre arte paleolítico, hemos intentado demostrar en este artículo las posibilidades que la historia social de las ciencias sociales de Pierre Bourdieu ofrece para la constitución de una historia crítica de la arqueología. Convertida en un instrumento de reflexión sobre la práctica arqueológica, dicha historia debe servir a los arqueólogos para comprender mejor la naturaleza de su propio trabajo. En este sentido, pensamos que se trata de un modelo que permite cumplir las expectativas de los historiadores de la arqueología con respecto al papel que su disciplina debe jugar en el conjunto de la ciencia: "Quiero sugerir que hay un lugar importante, de hecho es una necesidad acuciante, para una historiografía crítica de la disciplina que articule el pasado directamente con los intereses filosóficos y teóricos contemporáneos y futuros. En la medida en que esos intereses van a proporcionar un conocimiento más profundo de la naturaleza de la empresa arqueológica como vehículo para su continuo crecimiento y reformulación, la historiografía de la arqueología debería ser vista como un componente fundamental de lo que en la terminología de Clarke constituye un análisis interno de la disciplina." (Pinsky, 1989: 88).

Para finalizar, quisiéramos concluir con un texto de Pierre Bourdieu que de alguna manera resume también nuestro proyecto,

"Me ha parecido particularmente necesario someter a la ciencia a un análisis histórico y so- 
ciológico que no tiene como objetivo relativizar el conocimiento científico reduciéndolo a sus condiciones históricas [...] sino que pretende, al contrario, permitir a aquellos que hacen la ciencia comprender mejor los mecanismos sociales que orientan la práctica científica y convertirse de este modo en "señores y poseedores" no sólo de la "naturaleza", de acuerdo con la vieja ambición cartesiana, sino también [...] del mundo social en el que se produce el conocimiento de la naturaleza" (Bourdieu, 2001: 8).

\section{AGRADECIMIENTOS}

Este trabajo es el resultado de una línea de investigación que hemos desarrollado durante varios años. En este sentido, nos hemos beneficiado de los consejos de compañeros y amigos a los que quisiéramos expresar nuestro más sincero agradecimiento. El análisis de la historia social de las ciencias sociales debe mucho a los comentarios de Luis Enrique Alonso (Universidad Autónoma), Francisco Vázquez (Universidad de Cádiz) y de José Luis Moreno Pestaña (Universidad de Jaén), tres de los grandes conocedores de la obra de Pierre Bourdieu en nuestro país. Nuestro trabajo sobre el arte paleolítico se ha beneficiado de los comentarios y críticas de varios colegas: Denis Vialou (Muséum National d'Histoire Naturelle), Randall White (University of New York), Margaret Conkey (University of California, Berkeley), Lawrence G. Straus (University of New Mexico), Margarita Díaz-Andreu (University of Durham) y Larry Shiner (University of Springfield). Por último, nuestro punto de vista sobre la historia de la arqueología se ha enriquecido notablemente gracias a las aportaciones de un grupo de historiadores con los que compartimos muchas ideas: Marc-Antoine Kaeser (Musée National Suisse), Wiktor Stozckowski (École des Hautes Etudes en Sciences Sociales), Nathan Schlanger (Université de Paris I), Gloria Mora (Universidad Autónoma de Madrid), Víctor M. Fernández (Universidad Complutense de Madrid) y Alain Schnapp (Université de Paris I).

\section{BIBLIOGRAFÍA}

Alonso, L. E.; Martín Criado, E. y Moreno Pestaña, J. L. (eds.): Pierre Bourdieu: las herramientas del sociólogo, Madrid, Fundamentos, 2004.

ATKINSON, J. Y BANKS, I \& O'SULLIVAN, J.: Nationalism and archaeology, Glasgow, Cruithne Press, 1996.

Bahn, P. G.: Dictionary of Archaeology., Glasgow, HarpersCollins Publishers, 1992.

BAHN, P. G.: "Cave art without the caves", Antiquity ,69 (263), 1995, pp. 231- 237.

Barandiarán, I.: Arte mueble del Paleolítico Cantábrico, Zaragoza, Universidad de Zaragoza, 1972

BARTON, C. M.; ClaRk, G. A. y COHEN, A. E.: "Art as information: explaining Upper Palaeolithic art in western Europe", World Archaeology 26 (2), 1994, pp. 185-207.

BENDER, S. J.: "The History of Archaeology and the Liberal Arts CurricuIum". En: Andrew L. Christenson (ed.). 1989: Tracing Archaeology's past. The historiography of Archaeology, Carbondale and Edwardsville, Southern Illinois University Press, 1989, pp. 54- 61.

BLOOR, D.: "Wittgenstein and Manheim on the Sociology of Mathematics". En: Harry M. Collins (ed.): Sociology of Scientifi Knowledge. A source Book, Bath, Bath University Press, 1988, pp. 39- 57.

Bourdieu, P.: "Célibat et condition paysanne", Etudes Rurales, 5- 6, 1962, pp. 32-135.

BouRdieu, P.: "La spécificité du champ scientifique et les conditions sociales du progrès de la raison". Sociologie et Sociétés, 7 (1), 1975, pp. 91-117.

Bourdieu, P.: "Le champ scientifique". En : P. BouRdieu: Intelectuales, política y poder, Buenos Aires, Eudeba, 1999, pp. 75- 110.

Bourdieu, P.: Le sens pratique., Paris, Les éditions de Minuit, 1980.

Bourdieu, P.: Questions de sociologie, Paris, Les Editions de Minuit, $1980 b$.

BOURDIEU, P.: Leçon sur la leçon (Leçon inaugurale prononcée au Collège de France le vendredi 23 avril 1982), Paris, Les Editions de Minuit, 1982.

BouRdieu, P.: Choses dites, Paris, Les Editions de Minuit, 1987.

BOURDIEU, P.: Les règles de l'art. Genèse et structure du champ littéraire, Paris, Seuil (Collection Libre Examen). 1992.

Bourdieu, P: Réponses. Pour une anthropologie réflexive, Paris, Seuil, $1992 b$.
Bourdieu, P: Raison pratiques. Sur la théorie de l'action, Paris, Seuil, 1994

Bourdieu, P.: "La cause de la science. Comment I'histoire sociale des sciences sociales peut servir le progrès de ces sciences" (1995) .En: P. BOURDIEU: Intelectuales, politica y poder, Buenos Aires, Eudeba, 1999, pp. 111- 127.

Bourdieu, P.: Méditations pascaliennes, Paris, Seuil (Collection Liber), 1997.

Bourdieu, P.: La domination masculine, Paris, Seuil (Collection Liber), 1998.

Bourdieu, P.: Les structures sociales de l'économie, Paris, Seuil (Collection Liber), 2000.

Bourdieu, P: Propos sur le champ politique, Lyon, Presses Universitaires de Lyon, 2000a.

Bourdieu, P.: Science de la science et réflexivité. Cours du Collège de France. 2000- 2001, Paris, Raisons d'Agir Éditions, 2001.

Bourdieu, P.: "L'objectivation du sujet de l'objectivation" (2004). En: J. Heilbron, R. Lenoir y G. Sapiro (eds.): Pour une histoire des sciences sociales. Hommage à Pierre Bourdieu. Paris, Fayard, 2004, pp. 1923.

Bourdieu, P.; Chamboredon, J. C. y PAsseron, J. C.: Le métier de sociologue Préalables épistémologiques, Paris, Mouton, 1964.

BRAdLEY, R.: Parietal art and the prehistory of atlantic Europe, London \& New York, Routledge, 1997.

Bray W., y Trump D.: A dictionary of Archaeology, The Penguin Press, London, 1970.

BRÉZILLON, M.: Dictionnaire de la préhistoire, Paris, Larousse, 1969.

Calhoun, C.; Lipuma, E. y Postone, M. (eds.): Bourdieu. Critical Perspectives, Cambridge, Polity Press, 1963.

CARTAILHAC, E.: "Les cavernes ornées de dessins. La grotte d'Altamira, Espagne". Mea culpa d'un sceptique. L'Anthropologie XIII, 1902, pp. 348- 354.

CHIRON, L: "La grotte Chabot, commune d'Aiguèze, Gard". Bulletin de la Société d'anthropologie de Lyon 8, 1892, pp. 96-97.

COYE, N.: La préhistoire en parole et en acte. Méthodes et enjeux de la pratique archéologique (1830-1950), Paris, L'Harmattan, 1997.

DELPORTE, H.: L'objet d'art préhistorique, Paris, Éditions de la Réunion des Musées Nationaux, 1981.

DELPORTE, H.: La imagen de los animales en el arte prehistórico (primera edición 1990), Madrid, Compañía Literaria. 1995. 
DE MoRTILlet, G.: Formation de la Nation Française. Textes-LinguistiquePalethnologie-Anthropologie, Paris, Félix Alcan, 1897.

D'ERRICO, F.: "Lecture technologique de l'art mobilier gravé. Nouvleees méthodes et premiers résultats sur les galets gravés de Rochedane", L'Anthropologie, 1988, 92-1, pp. 101. 122.

Díaz-AndREU, M.: Historia de la arqueología en España. Estudios, Madrid, Ediciones Clásicas, 2002

DiAz-AndREU, M. y CHAMPION, T: Nationalism and archaeology in Europe, Colorado, Westview Press: 1996.

Diaz-AndREU, M. y SoREnSEN, M. L.: Excavating women: a history of women in European archaeology, London, Routledge, 1998.

DreYFus, C.: L'évolution des mondes et des sociétés, Paris, Félix Alcan Éditeur, 1888.

DUPONT, E.: L'Homme pendant les ages de la pierre dans les environs de Dinant- sur-Meuse., Bruxelles, C. Muquardt Éditeur., 1872.

Evans, J.: Les Ages de la Pierre. Instruments, armes et ornements de la Grande- Bretagne (primera edición 1872), Paris, Germer Baillière, 1878

FAHNESTOCK, P. J.: "History and theoretical development: the importance of a critical historiography of archaeology", Archaeological Review From Cambridge 3, 1984, pp. 7-18.

FAlCONER, H.: "Works of art by primeval man in Europe" (primera edición 1864). En: Charles Murchinson (ed.): Palaentological memoirs and notes of the late Hugh Falconer. Vol II. Mastodon, Elephant Rhinoceros, Ossiferous Caves, Primeval Man and his cotemporaries, London, Robert Hardwicke, 1868, pp. 626-631.

FoucAuLt, M.: "Los intelectuales y el poder" (primera edición 1972). En: M. Foucault: Estrategias de poder. Obras esenciales. Volumen II. Barcelona, Paidós, 1999, pp. 106-115.

Gran-AYMERICH, Ė.: Naissance de l'archéologie moderne. 1798-1945, Paris, CNRS Éditions, 1998

GROENEN, M.: Pour une histoire de la préhistoire. Le paléolithique, Grenoble, Éditions Jérôme Million., 1994.

GUSTAFSSON, A.: "The history of archaeology: Good Archaeology as Bad History?" (1998). En: A. -C. Andersson, A. Gillberg, O. W. Jensen, H. Karlsson y M. V. Rolöf (eds.) The Kaleidoscopic Past. Proceedings of the 5th Nordic TAG Conference. Göteborg, 2- 5 April 1997. Göteborg. Göteborg University, pp. 285-293.

HÄRKE, H. (ed.): Archaeology, Ideology and Society. The German Experience, Frankfurt, Peter Land, 2000.

HORKHeImer, M. Y ADORno T. W.: Dialéctica de la llustración. Fragmentos filosóficos (primera edición 1944), Madrid, Trotta, 1994.

JonEs. S.: The archaeology of Ethnicity. Constructing identities in the past and the present, London and New York, Routledge, 1997.

KAESER, M. A.: L'univers du prehistorien. Science, foi et politique dans I'œuvre et la vie d'Edouard Desor (1811-1882), Paris, L'Harmattan, 2004.

KенOE, A. B.: The Land of Prehistory. A Critical History of American Archaeology, London, Routledge, 1998.

KoHL, P. L. Y FAWCET, C.: Nationalism, politics, and the practice of archaeology, Cambridge, Cambridge University Press, 1995.

KRISTELLER, O. P.: II sistema moderno delle arti (primera edición 1950), Firenze, Uniedit, 1977.

LAHIRE, B. (ed.): Le travail sociologique de Pierre Bourdieu. Dettes et critiques, Paris, La Découverte., 1999.

LAMIng-EMPERAIRE, A.: La signification de l'art rupestre paléolithique, Paris, Picard. 1962.

LARTE, E. y CHRISTY, H.: "Sur des figures d'animaux gravées ou sculptées et autres produits d'art et d'industrie rapportables aux temps primordiaux de la période humaine" (primera edición 1864). En: N. Richard: L'invention de la préhistoire. Une anthologie, Anglaterre, Pocket, 1992, pp. 245- 285.

LANE, J.: Pierre Bourdieu. A Critical Introduction., London, Pluto, 2000.

LÉrol-Gourhan, A.: Préhistoire de l'art occidental, Paris, Éditions d'art Lucien Mazenod, 1971

LORBLANCHET, M.: Les grottes ornées de la préhistoire. Nouveaux regards, Paris, Édition Errance, 1995.

LUBвock, J.: L'Homme préhistorique. Étudié d'après les monuments re- trouvés dans les différentes parties du monde suivi d'une description comparée des mœurs des sauvages modernes (primera edición 1865), Paris, Librairie Germer Baillière, 1876.

Luввоск, J.: The Origin of Civilization and the Primitive Condition of Man, London, Longman, 1870.

LuKÁCS, G.: Historia y conciencia de clase, Madrid, Sarpe, 1923.

MAINE, H.: Ancien Law., London, J. Murray. 1863.

MCLENNAN, J. M.: Primitive Marriage: an Inquiry into the Origin of the Form of Capture in Marriage Ceremonies, Edinburgh, Black, 1865.

MetzZER, D. J.: "A question of relevance". En: Andrew L. Christenson (ed.) Tracing Archaeology's past. The historiography of Archaeology, Carbondale and Edwardsville, Southern Illinois University Press, 1989, pp. 5-20.

Menëndez, M.; Jimeno, A. y Fernández, V. M.: Diccionario de la Prehistoria, Madrid, Alianza Editorial, 1997.

Moreno PeStaña, J. L.: "Cuerpo, género y clase en Pierre Bourdieu". En: L. E. Alonso, E. Martín Criado, J. L. Moreno Pestaña (eds.): Pierre Bourdieu: las herramientas del sociólogo, Madrid, Fundamentos, 2004, pp. 143-183.

Morgan, L. H.: Ancient Society: Researches in the Lines of Human Progress from Savagery trough Barbarism to Civilization, New Yor, Holt, 1877.

Moro AbadiA, O.: "The War Against the Whigs. Victory...or Defeat?". Comunicación presentada en International Conference "Histories of Archaeology: Archives, Ancestors, Practices", AREA (Archives of European Archaeology). Göteborg, 17- 19 June 2004.

Moro Abadía, O. y González Morales, M. R.: "L'art bourgeois de la fin du XIXe siècle face à l'art mobilier Paléolithique". L'Anthropologie 107, 2003, pp. 455-470.

Moro AbadiA, O. Y GonzÁlez Morales, M. R.: "Towards a Genealogy of the Concept of "Paleolithic Mobiliary Art". Journal of Anthropological Research, 60 (3), 2004, pp. 321-339.

MoRTILLE, G.: Le préhistorique (primera edición 1883), Reinwald, Paris, pp. 411- 422. En: N. Richard: L'invention de la préhistoire. Une anthologie, Anglaterre, Pocket, 1992, pp. 288- 298.

MortILlet, G.: Formation de la Nation Française. Textes-LinguistiquePalethnologie-Anthropologie, Paris, Félix Alcan., 1897.

MOUNIER, P. 2001: Pierre Bourdieu. Une introduction. La Découverte. Paris.

MuRraY, T. (ed.): Enclyclopedia of Archaeology. The Great Archaeologist. II Volumes, Santa Barbara, ABC-CLIO, 1999

MURRAY, T. (ed.): Encyclopedia of Archaeology. History and Discoveries. III Volumes, Santa Barbara, ABC-CLIO, 2001.

MuRRAY, T: "Epilogue: why the history of archaeology matters", Antiquity 76 (291), 2002, pp. 234-238.

PINSKY, V.: "Introduction: historical foundations". En: V. Pinsky; A. Wylie (ed.): Critical traditions in contemporary archaeology. Essays in the philosophy, history and socio- politics of archaeology, Cambridge, Cambridge University Press, 1989, pp. 51-54.

PINSKY, V.: "Commentary: a critical role for the history of archaeology". En: V. Pinsky; A: Wylie (ed.) Critical traditions in contemporary archaeology. Essays in the philosophy, history and socio- politics of archaeology, Cambridge, Cambridge University Press, 1989, pp. 88-91.

Quiroga, F. y Torres CAmpos, D.: "La cueva de Altamira". Boletín de la Institución Libre de Enseñanza, 90, 1880, pp. 161-163.

RICHARD, N.: La préhistoire en France dans la seconde moitié du dix- neuvième siècle (1859- 1904). Thèse de doctorat sous la direction de Mme. C. Salomon- Bayet (Paris I), 1990.

RICHARD, N.: "Nouvelles perspectives de l'histoire de la préhistoire". Bulletin de la Société Préhistorique Française, 90 (1-2), 1993, pp. 11-12.

RoBerTsHAw, P. (ed.): A History of African Archaeology, London, J. Currey, 1990.

SABLOFF, J. A.: "Analyzing Recent Trends in American Archaeology from a Historical Perspective". En: Andrew L. Christenson (ed.): Tracing Archaeology's past. The historiography of Archaeology, Carbondale and Edwardsville, Southern Illinois University Press, 1989, pp. 34-40.

SAUTUOLA. M.: Breves apuntes sobre algunos objetos históricos de la provincia de Santander, Santander, Telesforo Martínez., 1880 
SAUVET, G. y WLODARCZYK, A.: "Elements d'une grammaire formelle de l'art pariétal paléolithique". L'Anthropologie, 99 2-3, 1995, pp. 193211.

SCHLANGER, N.: "Ancestral Archives: Explorations in the History of Archaeology". Antiquity, 76 (291), 2002, pp. 127-131.

Schnapp, A.: La conquête du passé. Aux origines de l'archéologie, Paris, Éditions Carré, 2003.

SHINER, L.: The Invention of Art. A Cultural History, Chicago, The University of Chicago Press, 2001.

SieVeKING, A.: The Cave Artist, London, Thames and Hudson, 1979.

SIlberman, N.: Between Past and Present. Archaeology, Ideology and Nationalism in the Modern Middle East, New York, Henry Holt and Company, 1989.

TRIGGER, B. G.: "Writing the History of Archaeology; A Survey of Trends". En: G. W. Stocking Jr. (ed.) Objects and Others: Essays on Museums and Material Culture. History of Anthropology, n. ${ }^{0}$, Madison, University of Wisconsin Press, 1985, pp. 218-235

TRIGGER, B. G.: Historia del pensamiento arqueológico (primera edición 1989), Barcelona, Crítica, 1992.

TRIGGER, B. G.: "Historiography". En: Tim Murray (ed.): Encyclopedia of
Archaeology. History and Discoveries. Volume II, Santa Bárbara ABC CLIO, 2001, pp. 630-639.

TYLOR, E. B.: Primitive Culture. Researches into the development of mythology, philosophy, religion, language, art, and custom, London, John Murray, 1871.

Ucko P. J. y Rosenfeld A.: Paleolithic Cave Art, London, World University Library, 1967.

VÁzouEz, F.: "¿Ortodoxia o reforma del entendimiento? La doble insolencia de Pierre Bourdieu. Excurso sobre las reflexividad". En: L. E. Alonso, E. Martín Criado, J. L. Moreno Pestaña (eds.): Pierre Bourdieu: Ias herramientas del sociólogo, Madrid, Fundamentos, 2004, pp. 351-373.

VIALOU, D.: "Problématique de l'interprétation de l'art paléolithique". Rivista di Scienze Preistoriche, XLIX, 1998, pp. 267-281.

VIALOU, D.: "Architecture de l'art pariétal paléolithique". Actes du colloque 8. 2. Congrès de I'UISPP, Liège 2001, Liège, pp. 7-14.

WhiteHOUSE, R. D.: The Macmillan dictionary of archaeology, London, MacMillan Press, 1983.

WILSON, D.: Prehistoric Man. Researches into the Origin of civilization in the Old and the New World (primera edición 1862), London, MacMillan and Co., London, 1865. 